\title{
The influence of cultural framing on play in the trust game: a Maasai example \\ Lee Cronk*
}

Department of Anthropology and Center for Human Evolutionary Studies, Rutgers University, New Brunswick, NJ 08901-1414, USA

Initial receipt 25 July 2006; final revision received 8 May 2007

\begin{abstract}
The effects of cultural framing on behavior in experimental games were explored with a trust game and the Maasai concept of osotua. Maasai use the term osotua to refer to gift-giving relationships based on obligation, need, respect, and restraint. In the trust game, the first player is given money and an opportunity to give any portion of it to the second player. The amount given is then multiplied by the experimenter, and the second player has an opportunity to give any amount back to the first player. Fifty trust games were played by Maasai men at a field site in north central Kenya. Half of the games were played without deliberate framing, and half were framed with the statement, "This is an osotua game." Compared to games with no deliberate framing, those played within the osotua rhetorical frame were associated with lower transfers by both players and with lower expected returns on the part of the first players. Osotua rhetorical framing is also associated with a negative correlation between amounts given by the first player and amounts returned by the second. These results have implications both for the experimental game method and for our understanding of the relationship between culture and behavior.
\end{abstract}

(C) 2007 Elsevier Inc. All rights reserved.

Keywords: Cooperation; Trust game; Maasai; Experimental games; Framing effects; Culture

\section{Introduction}

Experimental economic games have become a popular and important tool for the exploration of such topics as fairness, trust, and cooperation across human societies. The result is a well-established and widely cited new field of empirical research with important theoretical implications (e.g., Gintis, Bowles, Boyd, \& Fehr, 2005; Henrich et al., 2004). Although one of the main motivations behind the use of such games has been a desire to explore the influence of culture on behavior (e.g., Henrich, 2000), both critical and sympathetic commentators (e.g., Chibnik, 2005; Hagen \& Hammerstein, 2006) have decried these studies' lack of attention to culture, in general, and to rhetorical framing, in particular. This article addresses this problem by examining

\footnotetext{
is Rutgers' Center for Human Evolutionary Studies (CHES) provided funding for the 2005 field season. The CHES, the National Science Foundation, the Population Council, a Fulbright grant, Texas A\&M University, and Rutgers University provided funding for previous field seasons.

* Department of Anthropology, Rutgers University, 131 George St., New Brunswick, NJ 08901-1414, USA.

E-mail address: lcronk@anthropology.rutgers.edu.
}

the effects of locally salient rhetorical framing on how people play a trust game. This yields insights about the impact of rhetorical framing on such games, the relationship between culture and behavior, and the details of such specific rhetorical frame and the particular game used in this study.

The rhetorical frame in question is the Maa concept of osotua. Maa is the language spoken by such large East African pastoralist groups as the Maasai and Samburu, as well as by a variety of neighboring peoples. This project was conducted in Mukogodo Division, Laikipia District, Kenya, where I have been doing ethnographic research and have been studying the Maa language since 1985 (Cronk, 2004). Maa speakers use the term osotua to refer to certain kinds of gift-giving relationships. Partners in such relationships are called isotuatin. The basic method was to run equal numbers of trust games without any deliberate rhetorical framing and with osotua as a rhetorical frame.

\section{Qualitative background: the osotua concept}

Osotua's literal meaning is "umbilical cord," making it a particularly evocative bit of rhetoric for the discussion of 
gift-giving relationships. Economic anthropologists (e.g., Bird-David, 1990; Gudeman, 1986) have suggested that people understand their societies' economies through central metaphors, and osotua may be regarded as the central metaphor of Maasai ethnoeconomics. Osotua's centrality to Maasai life was noticed by western Bible translators, who evoked the idea of a bond between God and people by translating "testament" as "osotua." Despite the great fame of the Maasai and the important role that osotua plays in Maasai economic life, osotua has received little attention from ethnographers. Most mentions of osotua in the existing ethnographic literature on Maa-speaking peoples are limited to brief descriptions of isotuatin as bond friends, stocksharing partners, and stock friends (e.g., Spencer, 1965, pp. 27, 59; Spencer, 1988, p. 39). Hollis (1905/1971, pp. 289, 321-322) translates osotua as "peace," but more in the sense of a peace treaty or a peaceful bond between former enemies, rather than a general state of peacefulness, which is referred to by another term (eseriani). Jacobs $(1965$, p. 210) makes the important observation that because osotua, in its literal sense, refers only to a human umbilical cord, its metaphorical use emphasizes the humanness of such relationships (according to Jacobs, a nonhuman umbilical cord is called osarikoma).

In light of the limitations of previous qualitative research on osotua, I began this project in early June 2005 by conducting semistructured interviews with 10 men living around my field site. Participants in these interviews ranged in age from 25 to 73 years, with a mean age of 45 years. Each man was paid 100 Kenyan shillings (about US\$1.33) for his participation in the study. All interviews were conducted in Maa and recorded on audiotape. Interviews were guided by a list of 15 open-ended questions on osotua. These included questions about the meanings of the terms osotua and isotuatin, the ways in which people become isotuatin, how long such relationships last, what isotuatin are expected to do for each other, what kinds of people become involved in osotua relationships, whether anything can end an osotua relationship, and what happens when isotuatin die. Informal discussions with these same men about the results of trust games (see below) also improve my understanding of osotua.

The 10 interviewees displayed a very high degree of consensus regarding the major features of osotua relationships, which I will summarize briefly. Osotua relationships are started in many ways, but they usually begin with a request for a gift or favor. Such requests arise from genuine need and are limited to the amount actually needed. Gifts given in response to such requests are given freely (pesho) and from the heart (ltau) but, like the requests, are limited to what is actually needed. Because the economy is based on livestock, many osotua gifts take that form, but virtually any good or service may serve as an osotua gift. Once osotua is established, it is pervasive in the sense that one cannot get away from it. Osotua is also eternal. Once established, it cannot be destroyed, even if individuals who established the relationship die. In that case, it is passed on to their children (see also Spencer, 1965, p. 59). Osotua does not follow a schedule. It will not go away even if much time passes between gifts. Although osotua involves a reciprocal obligation to help if asked to do so, actual osotua gifts are not necessarily reciprocal or even roughly equal over long periods of time. The flow of goods and services in a particular relationship might be mostly or entirely one way, if that is where the need is greatest. Not all gift giving involves or results in osotua. For example, some gift giving results instead in debt (sile). Osotua and debt are not at all the same. While isotuatin have an obligation to help each other in time of need, this is not at all the same as the debt one has when one has been lent something and must pay it back (see also Spencer, 1965, p. 27). Going along with the idea that osotua gifts do not repay debt, osotua gifts are not payments at all, and it is inappropriate to use the verb "to pay" (alak) when referring to them. Osotua imbues respect (enkanyit), restraint, and a sense of responsibility in a way that non-osotua economic relationships do not. In the words of one interviewee, "keiroshi" ("It is heavy"). Osotua thus exemplifies a type of relationship that is widespread across human societies and in which short-term reciprocity and account keeping are considered inappropriate (Silk, 2003). Finally, osotua is very, very important. Indeed, during the interviews, I was struck by the fact that, after many years of asking questions about things of great interest to me but of little importance to my interviewees, I was finally asking them about something that they themselves considered very significant.

Disagreement among interviewees was minimal. The only significant disagreements were in response to a question about whether anything could end or "cut" (adung) osotua. Most said no, but one said that a lie — whether told to elicit a gift or a gift larger than what is actually needed, or in response to a request from an osotua partner-would end the relationship. However, he also made clear that such behavior was unthinkable. Osotua partners are expected to request only what they need and to give what is needed (although no more than that) if they are able to do so. Another suggested that only a war could end an osotua relationship.

One man illustrated many of osotua's main features through a story about his own family. Some decades ago, his ancestor Kimbai was killed by two men from an enemy group. One of Kimbai's killers then removed his warrior's belt (ntore) and wore it as a trophy. After the fight, the killers visited a man from another local group and asked him for food, lodging, and medicine to treat their wounds. Unbeknown to the visitors, their host and Kimbai were isotuatin. That man's wife recognized Kimbai's belt and deduced that the visitors had killed him. She and her husband slaughtered a sheep for fat to feed the visitors, poisoned the fat, killed the two visitors, and thus avenged Kimbai's death. This revenge killing was a form of osotua gift to the dead Kimbai and, by extension, to his survivors. The belt was then returned to Kimbai's grandfather, and a bond of osotua has existed between the two families ever since. 


\section{Methods}

Different experimental economic games are suited to the exploration of different aspects of behavior. For this study, I chose the trust game (also called the investment game; Berg, Dickhaut, \& McCabe, 1995) because it better resembled the give-and-take pattern of osotua relationships than, say, the more commonly used dictator or ultimatum games. Two slightly different versions of the trust game have been used by researchers. Both are two-player games in which the first player is given some amount of money and allowed to give any portion of it, or none at all, to the second player and to keep whatever remains. The experimenter then triples the amount given to the second player, who can return any portion of his enlarged stake to the first player, keeping whatever remains. In the simplest version of the game, only the first player receives an initial endowment. A slight modification of the game is to give both players equal initial endowments (Barr, 2004; Lesorogol, unpublished data). Endowing the second player as well as the first player is intended to reduce the chance that the amount the first player decides to give to the second player reflects notions of fairness and to increase the chance that it reflects feelings of trust. Because I was interested more in trust than in fairness, I used the version of the game in which both players are given an initial endowment. In the experimental game literature, the first player is often called the "investor" and the second player is called the "trustee," but for reasons that will become clear later in this article, I refer to them instead simply as Players 1 and 2.

I conducted the games in three phases. On June 20, 50 men acted as Player 1 in a building near the divisional headquarters of Don Dol. After playing, those men received whatever portion of their stake that they did not give to Player 2, as well as a receipt identifying them by a code name (one of the two-letter abbreviations of the 50 states of the United States). They were instructed to bring their receipt back 2 days later to receive whatever Player 2 had decided to give them. On June 21, my assistants and I drove to the town of Il Polei, about $15 \mathrm{~km}$ from Don Dol, and had 50 men play the role of Player 2. People in both areas are Maa speakers who share an understanding of osotua, but they have little interaction, particularly from one day to the next, such that anonymity between those in the position of Player 1 and those in the position of Player 2 was likely to be as complete as it possibly can be in a field setting. On June 22, we returned to Don Dol and paid the men who had played the role of Player 1 whatever their corresponding Player 2 had decided to give them.

The games were played using coins worth 10 Kenyan shillings each. Upon arrival, each player went through a brief individual training session about how the game works, following a script similar to those used in other field studies using the trust game (e.g., Barr, 2004; Ensminger, 2000; Lesorogol, unpublished data). Instructions were given in Maa by my field assistant while I used coins to demonstrate the consequences of different allocations and, while training those in the role of Player 2, showed what Player 1 had done the day before. We used small wooden boards, one in front of the player and one on the other side of the table, to display the amounts held by the two players. Players were told only that the other player was a man from the Mukogodo area who was also Maasai. The training session included a series of questions designed to test participants' understanding of the game. Specifically, a series of hypothetical transfers ${ }^{1}$ from Player 1 was shown, and participants were asked what the consequences of those transfers would be for Player 1's remaining cash and for Player 2's subsequent endowment. Although literacy rates are low among adult men in the Mukogodo area, people there do use money routinely and are comfortable doing basic arithmetic operations involving cash and coins. The fact that everything was represented using actual coins aided participants' understanding of the game. As a result of these careful efforts to train participants, most caught on to the game very quickly, and my field assistant and I were confident that all understood the game before they actually played it. After they were finished playing, they were instructed to leave the premises and not to discuss the game with anyone else. I hired two men to ensure that men waiting to play remained out of earshot of the buildings in which the games were being played and that players left the area as soon as they had finished playing. Each player began with a stake of 100 Kenyan shillings ( US\$1.33). That is about a day's wage and also matches the amount used by Lesorogol (unpublished data) when playing the trust game with the Maa-speaking Samburu of northern Kenya (see also Lesorogol, 2005). We also asked those in the role of Player 1 how much they expected to receive back from Player 2. Finally, all players were given a soft drink and a packet of snuff to thank them for their participation.

On both days of game playing, the first 25 players were instructed on how to play the game without any deliberate rhetorical framing. The second 25 players were given the same instructions with the additional framing sentence, "This is an osotua game" (Nena enkiguran o osotua). Because the order in which participants played the game was determined solely by when they happened to arrive at the study location, their assignment to one of the two treatments was essentially random. In any particular game, the presence or the absence of the osotua rhetorical frame was the same for both Players 1 and 2.

In addition to game-playing data, we asked each participant for his age, the name of his age set (a common

\footnotetext{
${ }^{1}$ In accordance with a preference in the experimental economic game literature for neutral language, I use the noun "transfer" to refer to the amount given by one player to another. However, the verb "transfer" would be inappropriate because the Maa word for "transfer" (aigutie) means "to place elsewhere" (i.e., to move something from one place to another; Mol, 1996, p. 97; see also Mol, 1979, p. 161). When running games, we use the Maa word aisho, meaning "to give." Therefore, when a verb is called for, I use "give."
} 


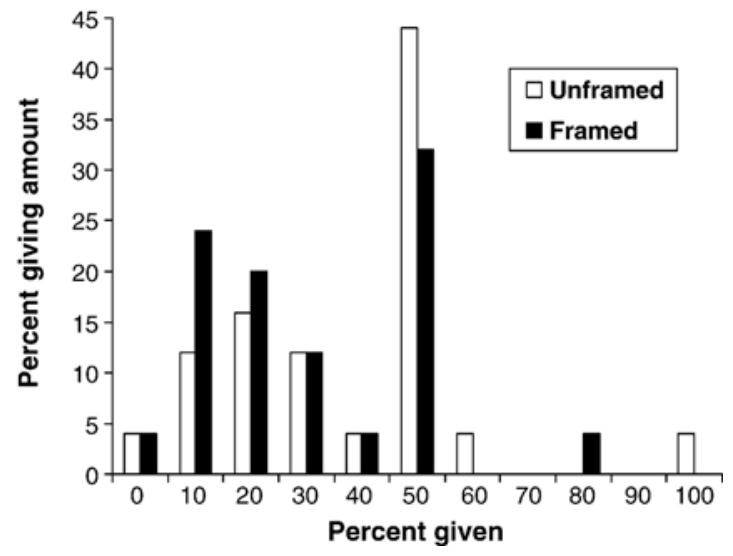

Fig. 1. Percentage of player's stake given to the other player, Player 1.

feature of East African pastoralist societies), and his number of wives (a rough proxy for wealth that is considered more polite than questions about, say, livestock holdings). The mean ages of players in a framed condition and in an unframed condition were very close [unframed $=34.34$, framed $=35.38 ; t_{98}=-0.382, p>.7$ (two tailed)], as were the mean numbers of wives [unframed $=0.92$, framed $=0.96 ; t_{98}=$ $-0.332, p>.7$ (two tailed)]. Furthermore, neither of those variables had any strong or statistically significant correlations with either amounts given or expected returns. For these reasons, neither age nor number of wives is considered in the analyses presented below.

\section{Results}

SPSS version 11.5.1 was used to compare mean transfers and mean expected returns in the unframed condition and in the framed condition. Both nonparametric (Mann-Whitney $U$ ) and parametric (Student's $t$ ) tests were used because valid arguments can be made for both. Because distributions are not normal, assumptions of parametric statistics are not met and so a nonparametric test may be more appropriate. On the other hand, $t$ test is robust when faced with such departures from normality and has often been used in the existing literature on experimental games.

Mean transfers and mean expected returns are all lower in the osotua framed condition, and most of those differences reach conventional levels of statistical significance. Considering all transfers together as proportions of what each player had available, the osotua rhetorical framing is associated with lower amounts (in mean \pm S.D.) [unframed $=$ $35.3 \pm 19.11$, framed $=28.2 \pm 16.11 ; t_{98}=2.014, p=.024$ (one tailed); Mann-Whitney $U=962.5, Z=-1.995, p=.023$ (one tailed)]. Transfers by Player 1 considered alone tended to be lower in the framed condition than in the unframed condition (Fig. 1), although this difference did not reach conventional levels of statistical significance [unframed $=38.00 \pm 21.60$, framed $=30.80 \pm 19.98 ; t_{48}=1.223, p=.11$ (one tailed); MannWhitney $U=249.50, Z=-1.266, p=.10$ (one tailed)]. Trans- fers by Player 2, considered as proportions of the total amounts available to those players, were also lower in the framed condition than in the unframed condition [Fig. 2; unframed $=32.50 \pm 16.23$, framed $=25.5 \pm 10.76 ; t_{48}=1.808$, $p=.039$ (one tailed); Mann-Whitney $U=230.0, Z=-1.605$, $p=.054$ (one tailed)]. As with transfers, osotua framing is associated with lowered expectations (unframed $=89.6 \pm$ 60.59 , framed $=64.0 \pm 50.00)-\mathrm{a}$ difference that reaches conventional levels of statistical significance using a nonparametric test [Mann-Whitney $U=225.0, Z=-1.767$, $p=.039$ (one tailed)] and approaches them using a parametric test $\left[t_{48}=1.629, p=.055\right.$ (one tailed) $]$.

Differences between the unframed condition and the framed condition also exist in the relationships between amounts given and expectations. In the unframed condition only, Player 1's expectations are significantly associated with Player 1's own transfers: Those who give more expect more in return (Fig. 3). However, it is only in the framed condition that the amount Player 2 returned is significantly associated with the amount given by Player 1 (Fig. 4). Specifically, in the framed condition, lower transfers from Player 1 are associated with higher returns from Player 2.

The semistructured interviews preceded the games by 2 weeks. Five of the 10 individuals who participated in the interviews also took part in the games, all as Player 1. The experience of having been interviewed about osotua might have constituted a demand characteristic, influencing the game play of these individuals and enhancing the effect of osotua framing. To examine this possibility, I reran the analyses without these five individuals. However, despite the reduction in sample size, removing interviewees from the analysis makes the osotua framing effect even stronger. While the difference between mean transfers from Player 1 in both unframed and framed conditions was not statistically significant with all players included in calculations, it is significant when interviewees are removed [unframed $=39.2 \pm 21.25$, framed $=29.1 \pm 17.58 ; t_{43}=1.725$, $p=.046$ (one tailed); Mann-Whitney $U=182.0, Z=-1.656$, $p=.049$ (one tailed)]. Similarly, while differences between

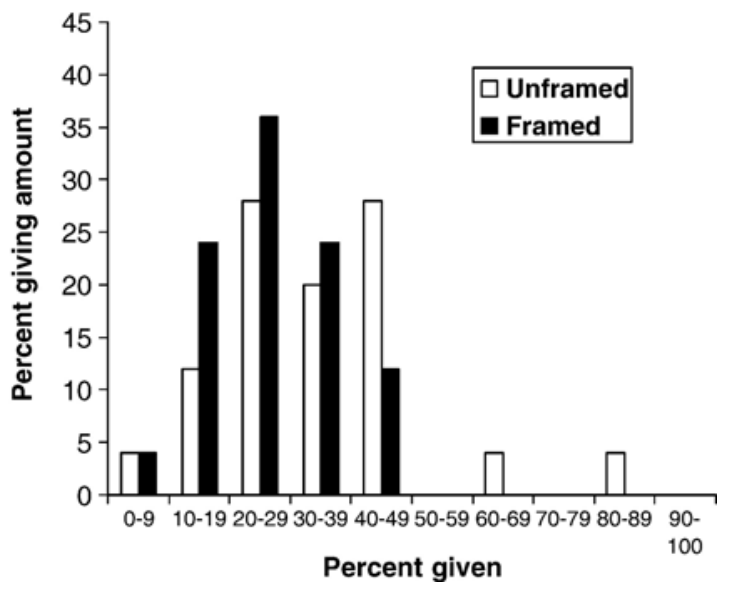

Fig. 2. Percentage given to Player 1 of the total amount available to Player 2. 


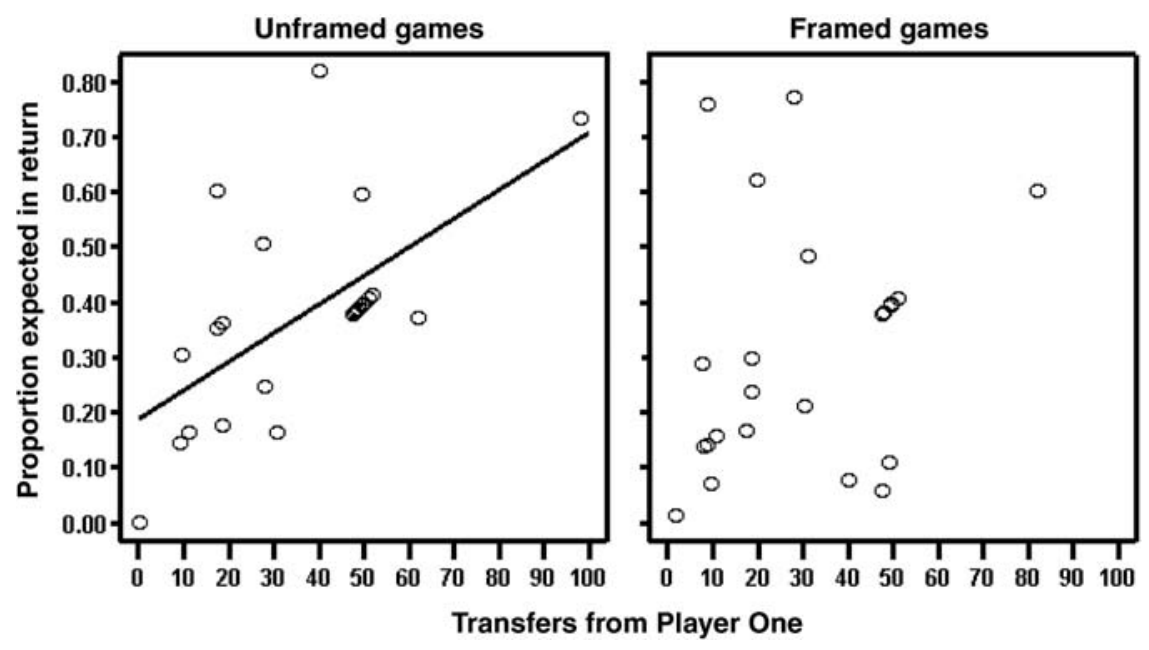

Fig. 3. Amounts given by Player 1 (horizontal axis) and the proportion of the total amount available to Player 2 expected in return (vertical axis), in both unframed (left) and framed (right) conditions. The standardized coefficient for an independent variable is statistically significant in the unframed condition $[\beta=$ $0.610, p<.001$ (one tailed), adjusted $\left.R^{2}=.35\right]$, but not in the framed condition $[\beta=0.272, p=0.095$ (one-tailed)].

expected returns in the two conditions reached conventional levels of statistical significance using a nonparametric test, but not with a parametric test, excluding the interviewees makes the difference statistically significant using both types of test [unframed $=91.7 \pm 60.98$, framed $=58.1 \pm 43.77$; $t_{43}=2.093, p=.021$ (one tailed); Mann-Whitney $U=164.0$, $Z=-2.101, p=.018$ (one tailed)]. Thus, if there was any effect of the interviews on game playing, it was only to make the effects of osotua rhetorical framing more difficult, rather than easier, to detect.

\section{Discussion}

Compared to trust games played without any deliberate rhetorical framing, games played within the rhetorical frame of osotua are associated with lower amounts given by trust game players and expected in return. All differences between the framed condition and the unframed condition are in the same direction, and most reach conventional levels of statistical significance. A positive correlation exists between amounts given and amounts expected in return in games played without deliberate rhetorical framing, but not in games framed with the rhetoric of osotua. In osotua-framed games but not in unframed games, amounts given by Player 1 and amounts returned by Player 2 are negatively correlated. These results have implications both for the experimental game method and for our understanding of the relationship between culture and behavior.

\subsection{Framing and the experimental game method}

Framing, whether part of a researcher's intentions or spontaneously imported by participants, is of concern to

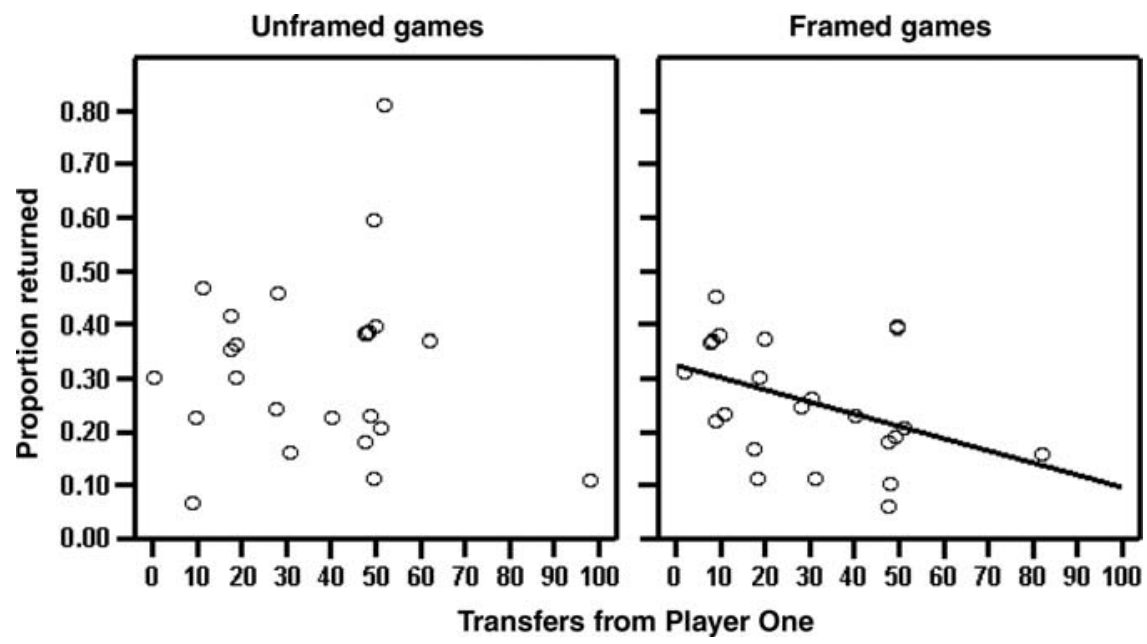

Fig. 4. Amounts given by Player 1 (horizontal axis) and the proportion of the total amount available to Player 2 returned to Player 1 (vertical axis), in both unframed (left) and framed (right) conditions. The standardized coefficient for an independent variable is statistically significant in the framed condition $[\beta=$ $-0.424, p=.018$ (one tailed), adjusted $\left.R^{2}=.144\right]$, but not in the unframed condition $(\beta=-0.042, p=.843$ ). 
anyone who works with experimental economic games. Despite the fact that all participants in this study had access to the osotua framework from their own experience as Maasai, those who played the game without deliberate osotua framing do not appear to have been strongly influenced by that cultural framework. Instead, the correlation between the amount that first players were given and how much they expected to receive in return (Fig. 3) suggests precisely the logic of trust, investment, and reciprocity that is usually assumed by researchers using the trust game. This is good news for those who would use this game to assess levels of trust among people in field settings.

On the other hand, this study demonstrates that when local cultural concepts are deliberately used to frame games, the result may be a noticeable shift in how they are played. Indeed, the shift may be strong enough that many of the assumptions and vocabulary usually associated with a particular game may become inappropriate. In the present case, for example, it would be wrong to interpret the lower transfers and expectations associated with osotua framing as an indication of reduced levels of trust among players. These findings, particularly those shown in Fig. 4, suggest instead that osotua framing shifts game play away from the logic of investment and towards the mutual obligation of isotuatin to respond to one another's genuine needs, but only with what is genuinely needed.

Osotua thus joins a small but growing list of local cultural frames that have been found to influence how people play experimental games. For example, Ensminger's (2000, 2004) Orma participants spontaneously used the Swahili word harambee to identify a public goods game. Orma and other Kenyans use harambee to refer to public goods projects and associated fund raising. Ensminger suggests that the participants were more comfortable and trusting while playing the public goods game because they associated it with a familiar institution. Tracer (2003) used local cultural traditions to explain unusual performances by Highland New Guinea participants in an ultimatum game. In an ultimatum game, the first player is given money and then may give any portion of it to the second player. If the second player accepts the offer, they each get to keep whatever they have. If the second player rejects the first player's offer, neither gets to keep anything. Tracer found that his participants tended to both make and reject high offers. While neither move is economically rational in a superficial sense, both are sensible moves in societies with competitive gift-giving systems, which are common in Highland New Guinea. In a laboratory study, Burnham, McCabe, and Smith (2000) found that referring to the other player as either "partner" or "opponent" had a significant effect on levels of trust and trustworthiness exhibited in an extensive form of the trust game.

Perhaps the best way to proceed is to combine experimental games with ethnographic research designed to reveal whatever cultural frames that might exist and to explore any such frames that the games themselves reveal. Any suspected framing effects can then be assessed using tests such as the one in this study. Other forms of framing should also be considered, both as potential problems for such projects and as opportunities for new insights. Perhaps, for example, the fact that most researchers are outsiders to the societies they study has the effect of framing experimental games played in field settings in a subtle way. Similarly, the use of cash may frame the game, putting players more in a frame of mind appropriate to market transactions than they would be if some other currency were used (Vohs, Mead, \& Goode, 2006). Further exploration of how such frameworks affect game play will both shed light on cultural variations and enhance the usefulness of the game method (Elliott and Hayward, 1998; Hagen and Hammerstein, 2006).

\subsection{Culture and the coordination of social behavior}

These results also demonstrate culture's role in coordinating social behavior. Although the relationship between culture and behavior is important to both evolutionary and nonevolutionary social theories, we have only a poor understanding of exactly how culture influences behavior (Cronk, 1999; D’Andrade, 1992, p. 23). One way to approach this issue is to look for variations in culture's influence on behavior. Cultural prescriptions that help coordinate social behavior can serve that purpose only if behavior conforms to them. The concept of osotua helps coordinate social behaviors among Maa speakers because there is a high degree of consensus about its major features and because it has a clear influence on actual behavior. This contrasts with a previously documented discrepancy between cultural rhetoric and behavior among the same people. Although Mukogodo Maasai say that they prefer sons, caregiver behavior favors daughters (Cronk, 1989, 1991, 2000, 2004). By espousing son favoritism, Mukogodo-area Maasai conform to a broader Maasai pattern, thus enhancing their claim (which is challenged by some Maa speakers) of being genuine Maasai. But because they are poor and of low status in a setting where polygyny and bridewealth lead to a correspondence between men's wealth and their marital success, behavioral favoritism toward daughters is more adaptive reproductively (Trivers \& Willard, 1973). The discrepancy between behavior and culture can persist in the realm of parenting because behaviors are performed by individuals with little need to coordinate their actions with anyone other than the children for whom they are caring. Osotua, on the other hand, is a social relationship requiring coordination between at least two individuals. Generalizing this contrast leads to a testable hypothesis: Behavior is more likely to correspond to cultural prescriptions when the latter help coordinate social behaviors than when they concern individual behaviors that do not need to be coordinated with others. This may, in turn, contribute to a theoretically predicted (Boyd \& Richerson, 2002; Henrich, 2004) and empirically observed (Henrich, et al., 2004; Henrich, et al., 2006) pattern in which learning dynamics generates equilibria in beliefs about norms of social 
interaction that are stable and widely shared within societies but which vary between them. Osotua may be the rhetorical manifestation of one such local equilibrium. The coordination of social behavior may have benefits at both individual and group levels. The relative importance of benefits at different levels in the evolution of such norms is an important topic for future research.

\section{Acknowledgments}

Thanks to K. Ole Leitiko for assistance in the field and to L. Ahearn, D. Fessler, B.L. Leech, R. Sosis, and two anonymous reviewers for helpful comments.

\section{References}

Barr, A. (2004). Kinship, familiarity, and trust: An experimental investigation. In J. Henrich, R. Boyd, S. Bowles, C. Camerer, E. Fehr, \& H. Gintis, (Eds.), Foundations of human sociality: Economic experiments and ethnographic evidence from fifteen small-scale societies (pp. 305-334). Oxford: Oxford University Press.

Berg, J., Dickhaut, J., \& McCabe, K. (1995). Trust, reciprocity, and social history. Games and Economic Behavior 10, 122-142.

Bird-David, N. (1990). The giving environment: Another perspective on the economic system of gatherer-hunters. Current Anthropology, 31, $183-196$.

Boyd, R., \& Richerson, P. (2002). Group beneficial norms can spread rapidly in a structured population. Journal of Theoretical Biology, 215, 287-296.

Burnham, T., McCabe, K., \& Smith, V. L. (2000). Friend-or-foe intentionality priming in an extensive form trust game. Journal of Economic Behavior \& Organization, 43, 57-73.

Chibnik, M. (2005). Experimental economics in anthropology: A critical assessment. American Ethnologist, 32, 198-209.

Cronk, L. (1989). Low socioeconomic status and female-biased parental investment: The Mukogodo example. American Anthropologist, 91, 414-429.

Cronk, L. (1991). Intention vs. behaviour in parental sex preferences among the Mukogodo of Kenya. Journal of Biosocial Science, 23, 229-240.

Cronk, L. (1999). That complex whole: Culture and the evolution of human behavior. Boulder: Westview Press.

Cronk, L. (2000). Female-biased parental investment and growth performance among the Mukogodo. In L. Cronk, W. Irons, \& N. Chagnon, (Eds.), Adaptation and human behavior: An anthropological perspective. Hawthorne, NY: Aldine de Gruyter.

Cronk, L. (2004). From Mukogodo to Maasai: Ethnicity and cultural change in Kenya. Boulder: Westview Press.

D'Andrade, R. G. (1992). Schemas and motivation. In R.G. D'Andrade, \& C. Strauss, (Eds.), Human motives and cultural models. Cambridge: Cambridge University Press.

Elliott, C. S., \& Hayward, D. M. (1998). The expanding definition of framing and its particular impact on economic experimentation. Journal of Socio-Economics, 27, 229-243.
Ensminger, J. (2000). Experimental economics in the bush: Why institutions matter. In C. Ménard (Ed.), Institutions, contracts and organizations: Perspectives from the new institutional economics. Cheltenham: Edward Elgar.

Ensminger, J. (2004). Market integration and fairness: Evidence from ultimatum, dictator, and public goods experiments in East Africa. In J. Henrich, R. Boyd, S. Bowles, C. Camerer, E. Fehr, \& H. Gintis, (Eds.), Foundations of human sociality: Economic experiments and ethnographic evidence from fifteen small-scale societies. Oxford: Oxford University Press.

Gintis H., Bowles S., Boyd R., \& Fehr E., (Eds.). (2005). Moral sentiments and material interests: The foundations of cooperation in economic life. Cambridge: MIT Press.

Gudeman, S. (1986). Economics as culture: Models and metaphors of livelihood. London: Routledge \& Kegan Paul.

Hagen, E. H., \& Hammerstein, P. (2006). Game theory and human evolution: A critique of some recent interpretations of experimental games. Theoretical Population Biology, 69, 339-348.

Henrich, J. (2000). Does culture matter in economic behavior? Ultimatum game bargaining among the Machiguenga Indians of the Peruvian Amazon. American Economic Review 90, 973-980.

Henrich, J. (2004). Cultural group selection, coevolutionary processes and large-scale cooperation. Journal of Economic Behavior and Organization, 53, 3-35.

Henrich J., Boyd R., Bowles S., Camerer C., Fehr E., \& Gintis H., (Eds.). (2004). Foundations of human sociality: Economic experiments and ethnographic evidence from fifteen small-scale societies. Oxford: Oxford University Press.

Henrich, J., McElreath, R., Barr, A., Ensminger, J., Barrett, C., Bolyanatz, A., Camilo Cardenas, Gruven, J., Gwako, E., Henrich, N., Lesorogol, C., Marlowe, F., Tracer, D., \& Ziken, J. (2006). Costly punishment across human societies. Science 312, 1767-1770.

Hollis, A. C. (1905/1971). The Masai: Their language and folklore. Freeport, NY: Books for Libraries Press.

Jacobs, A. (1965). The traditional political organization of the pastoral Masai. Doctoral dissertation, Oxford University, Oxford.

Lesorogol, C. K. (2005). Experiments and ethnography: Combining methods for better understanding of behavior and change. Current Anthropology, 46, 129-136.

Mol, F. (1979). Maa: A dictionary of the Maasai language and folklore. Nairobi: Marketing and Publishing.

Mol, F. (1996). Maasai language and culture dictionary. Lemek, Kenya: Maasai Centre; 1996.

Silk, J. B. (2003). Cooperation without counting: The puzzle of friendship. In P. Hammerstein (Ed.), The genetic and cultural evolution of cooperation (pp. 37-54). Cambridge: MIT Press. (Dahlem Workshop Report 90).

Spencer, P. (1965). The Samburu: A study of gerontocracy in a nomadic tribe. Berkeley: University of California Press.

Spencer, P. (1988). The Maasai of Matapato: A study of rituals of rebellion. Bloomington: Indiana University Press, in association with the International African Institute.

Tracer, D. (2003). Selfishness and fairness in economic and evolutionary perspective: An experimental economic study in Papua New Guinea Current Anthropology, 44, 432-438.

Trivers, R., \& Willard, D. (1973). Natural selection of parental ability to vary the sex-ratio of offspring. Science, 179, 90-92.

Vohs, K. D., Mead, N. L., \& Goode, M. R. (2006). The psychological consequences of money. Science, 314, 1154-1156. 